\title{
Relationship of the Heartbeat and Interbreath Interval Dynamics in Young and Elderly People
}

\author{
Wilson Bucaoto ${ }^{(1)}$ Artem Lenskiy ${ }^{(2)}$ \\ School of Electrical, Electronics and Communciation Engineering, Korea University of \\ Technology and Education \\ 1800 Chungjeol, Byeongcheon, Chonan, 330-708
}

E-mail: ${ }^{(1)}$ wilson.bucaoto@gmail.com, ${ }^{(2)}$ a.a.lensky@gmail.com

\begin{abstract}
Fluctuations in heartbeat and interbreath intervals have been confirmed to exhibit scale-invariance. This property can be used to examine the effect of gender, aging, health or disease of a patient from his heartbeat and respiration signals. Sophisticated methods of scale-invariant signal analysis are based on the estimation of multifractal spectrum of the scale exponent. In this study we used an algorithm to estimate multifractal spectrum to confirm the existence of nonlinear relationship between heartbeat and interbreath interval under supine resting condition for 20 young and 20 elderly people. Using two border and one peak scale exponents which are selected from each spectrum, we constructed a 3-dimensonal feature space. The distance and the angle of the line connecting features estimated from heartbeat and respiratory dynamics for each patient were calculated and compared in both age group. Results showed that long range dependency in heartbeat dynamics degrades in elderly people which results to a large difference between heartbeat and respiratory dynamics. Exponents for heartbeat and respiratory dynamics for young people have no significant difference reinforcing the evidence of a possible nonlinear relationship of heartbeat and respiratory dynamics for young people.
\end{abstract}

\section{Introduction}

Heartbeat dynamics and respiration dynamics are two of the most studied physiological processes primarily because of their clinical importance and complexity. These two processes are known to influence each other. The effect of breathing pattern on heart rate changes in main is called respiratory sinus arrhythmia (RSA) while the inverse is known as cardioventilatory coupling. Studies about heartbeat and respiration dynamics show that the two are linearly correlated such that the higher the respiratory rate, the smaller the higher the heart rate[1]. Respiratory period and tidal volume were shown also to influences RSA as well as the power spectrum of heart interbeat interval[2]. Effect of different breathing rates on heartbeat dynamics was also investigated and it was discovered that heart rate variability changes as breathing frequency, but average heart rate doesn't change [3].

While time-domain and frequency-domain analysis is useful in deriving the mean, variance and peaks in power spectrum of physiological signals, they are not suitable to be used for non-stationary signals such as heart beat and respiration dynamics. They also seldom uncover complex and fractal behaviour of the underlying processes.

A more sophisticated method of analyzing those signals involves using scale exponents. By estimating scaleexponents for physiological signals, the effects of gender, age and diseases can be studied.

There are numerous studies concerning the observation of the scaling properties to analyze heartbeat dynamics and respiration dynamics. In those studies it was shown that both processes demonstrate fractal characteristics.

Iyengar et al[4], conducted separate studies on the effect of aging in the alteration of scale-exponents in cardiac interbeat interval. They found a decrease in the complexity of Heart Rate Variability (HRV) for elderly subjects. Lenskiy et al found a multifractal behaviour of HRV in both young and elderly subjects, and confirmed that HRV is less correlated for elderly people.

Respiration dynamics was shown to have long-range correlation, although this correlation degrades in the case of elderly man. Peng et al. [5] computed the scaleexponents, or self-similarity parameter, of the inter-breath interval (IBI) signals from supine resting humans using the Detrended Fluctuation Analysis (DFA) and Fourier analysis. The DFA provided evidence for the fractal organization in physiologic human breathing cycle dynamics, and their degradation in elderly men. However, spectrum analysis did not show significant age or gender difference in the power spectrum exponents, due to the fact that the signals of IBI are nonstationary.

These findings were confirmed using a different method of fractal analysis [6], and in addition, demonstrated fractal fluctuations in two other respiratory parameters. The power 
law relationships in the Allan Factor, Fano factor and Dispersional Analysis plots reflected the long-range correlations among the fluctuations in the number of breaths and breathe amplitude, as well as the peak-to-peak breath interval. Whereas there was a fractal component of the fluctuations in all three of these parameters in 9 of 20 spontaneously breathing subjects, there were subjects in whom only the fluctuations in two, one or none of the parameters exhibited time-scale invariance, suggesting that they are controlled by different fractal processes.

A study on the relationship between the heartbeat dynamics and the dynamics of respiration under rest, exercises and increased atmospheric pressure showed no cross-correlation [7]. Using Holder exponent, a measure of the fluctuations related to the fractal dimension, they computed local fractal dimensions for the HRV and IBI variability and determined the cross-modal correlation across the population of subjects. They found a significant decrease in fractal dimension for both heartbeat and respiration variabilities with increasing levels of exercise. Although qualitatively similar, the heartbeat and respiration dynamics were not significantly correlated and are concluded to be statistically independent with regards to the response to exercise. Also, they did not see statistically significant effects of age on fractal dimension under any of the conditions. They recommended the search for non-linear interdependency.

One RSA study attempted to determine if the origin of fractal heart beat dynamics was due to the fractality of respiration.[8] Yamamoto et al. mentioned that HRV is better modeled as fractal process rather than sum of harmonics. The spectral exponent was determined using Coarse graining spectral analysis. They examined the cross-correlation of the spectral indices for the inverse power law spectra of Heart Rate Variability and Interbreath Interval variability. They concluded that the fractal behavior of the two processes were not correlated in any statistically significant way for subjects at rest, supporting the claim that the fractal component of heartbeat dynamics is not totally due to the respiratory modulation of heart rate. However, they suggested the possibility that respiratory influence on heart rate is nonlinear.

In this paper we are trying to investigate the possible nonlinear relationship of HRV and IBI in supine resting conditions and compare the variability among elderly and young people. We hypothesize that the multi-fractal spectrum (MFS) of both HRV and IBV are related to each other for one age or both group.

\section{Methods}

\subsection{Signal acquisition}

The researchers obtained two groups of heartbeat and respiration records, composed of 20 elderly (range 63-81) and 20 young (range 21-34) people, from an online database of physiological signals [9]. The subjects are rigorously screened healthy adults who laid supine for 120 min while watching a movie to maintain wakefulness. A set of records for a single subject contains the ECG with beat annotations and respiration signals.

Signals for the heartbeat interval for each subject were derived from the ECG beat annotations by subtracting the previous index to the current index and dividing by the sampling frequency. Conversely, respiratory annotations were not available, and so a Local Extrema Detection algorithm [10] was applied to extract the breathing points from the respiratory signals. The algorithm takes the signal and a delta threshold, which is the difference between a peak and its surrounding in order to identify it as an extrema. The function returns the indices and values of the local maxima and minima, which correspond to the exhalation and inhalation points. Effectively, intervals between two consecutive exhale points were extracted as the IBI signal. Misidentifications were addressed by visually inspecting the signal and ensuring that the peak threshold is optimally tuned such that it avoids identifying small bumps but at the same time correctly detecting true respiration peaks. The correction rules that we applied are based from a study [11] that also extracted interbreath interval from the same dataset. IBI were then derived as in heartbeat intervals. Preprocessing was also applied to remove the spikes in the signal.

\subsection{Multifractal spectrum estimation}

After extracting HRV and IBI variability for each subject, we estimated the multifractal spectrum. The applied algorithm for estimation of multifractal spectrum[12] is similar to the Detrended Fluctuation Analysis (DFA) [13] and multifractal DFA [14], however instead of polynomial subtraction, we apply a linear operator to suppress unwanted harmonics outside of the analysed frequency. The frequency range is proportional to the scale $l$. The estimation algorithm is summarized in the following four steps:

1. Apply sub-band filtering using a kernel obtained by taking a derivative of the Gaussian. The spread parameter in the Gaussian depends on the scale $l$;

2. Split the signal into $N_{l}$ overlapping sub-windows of length $2 l$ and calculate variance $\sigma_{k}^{2}(l)$ in each subwindow.

3. Calculating the the partition function as a sum of variances in each window powered by moment $q$ :

$$
\chi(q, l)=\sum_{k=1}^{N_{l}}\left[\sigma_{k}^{2}(l)\right]^{q}
$$

4. Estimating multfractal spectrum as follows: 


$$
\begin{array}{r}
f(q)=\lim _{l \rightarrow 0} \frac{\sum_{k=1}^{N_{l}} \mu_{k}(q, l) \ln \left(\mu_{k}(q, l)\right)}{\ln l}, \\
\alpha(q)=\lim _{l \rightarrow 0} \frac{\sum_{k=1}^{N_{l}} \mu_{k}(q, l) \ln \left(\sigma_{k}^{2}(l)\right)}{\ln l},
\end{array}
$$

where

$$
\mu_{k}(q, l)=\frac{\left[\sigma_{k}^{2}(l)\right]^{q}}{\chi(q, l)} .
$$

The analyzed process is divided into number of overlapping fragments each of them is treated as a standalone process. The estimated spectra are then averaged and the resulting spectrum is the one that characterize the whole process. Compared to DFA and MFDFA the above described algorithm is free of underestimation and overestimation problems due to linear operation of sub-filtering.

Using two border and one peak scale exponents which are selected from each spectrum, we constructed a 3dimensonal feature space. The border exponents correspond to values $q_{\min }=-10, q_{\max }=10$ and the peak exponent is obtained at $q_{\text {peak }}=0$, i.e.

$$
\boldsymbol{\alpha}^{T}=\left(\alpha_{\max }\left(q_{\text {min }}\right), \alpha_{\text {peak }}\left(q_{\text {peak }}\right), \alpha_{\min }\left(q_{\max }\right)\right)^{\mathrm{T}} .
$$

The distance and the angle of the line connecting features estimated from heartbeat and respiratory dynamics for each patient were calculated and compared in both age group.

\section{Results}

The separation of the heartbeat and respiration dynamics scale exponents for young and elderly is illustrated in Figure 1. In this picture we projected the scale exponents into a line and computed separate. To find projective axis defined by $w$, we applied fisher discrimenant approach. The constructed features $\boldsymbol{\alpha}$ are then projected on the axis $\boldsymbol{\alpha}^{T} w$. These projected points are then used to estimate parameres of Gaussian models for young and elderly using the values. The paremeters are estimated with maximumlikelihood method. For heartbeat dynamics, the separation of elderly and young patients is more evident than for the respiration dynamics, while exponents for the elderly are larger and more variable.

Figure 2 shows the 3-dimensional feature space constructed of $\boldsymbol{\alpha}$ for HRV and IBI for young and elderly people, as marked. We used connecting lines to compare the dynamics of heartbeat and respiration. To estimate MFS (2),(3) we used comparably short range of scales $L=\left\{l_{\min } \ldots l_{\max }\right\}$. The ranges $L$ were different for the HRV signals and IBI signals, since the latter is shorter compared to the former. We chose ranges that cover the same duration of time for cardiac and respiration signals. The ranges were selected to optimize the linear discriminant function estimated for values of $\boldsymbol{\alpha}$.

\section{Discussion}

The plot (fig. 2) hints for a non-linear relationship of the fractal properties of heartbeat and respiration dynamics for young subjects. The set of exponents for heartbeat and respiration dynamics for young are clustered in one region, with short distances, as compared to those for elderly patients. This indicates that the dimensions of the two sets of quantities, for young people can be regarded as 'the same' and are more likely to have a non-linear relationship. By virtue of the proximity in the dimensions and the Hausdorff dimension corollary[15], we infer that heartbeat and respiration dynamics can have one-to-one bi-Lipschitz mapping, while the dissociation and the consistent variability in the exponents for heartbeat and respiration dynamics in elderly patients provides no evidence for any nonlinear cardiorespiratory variability relationship.

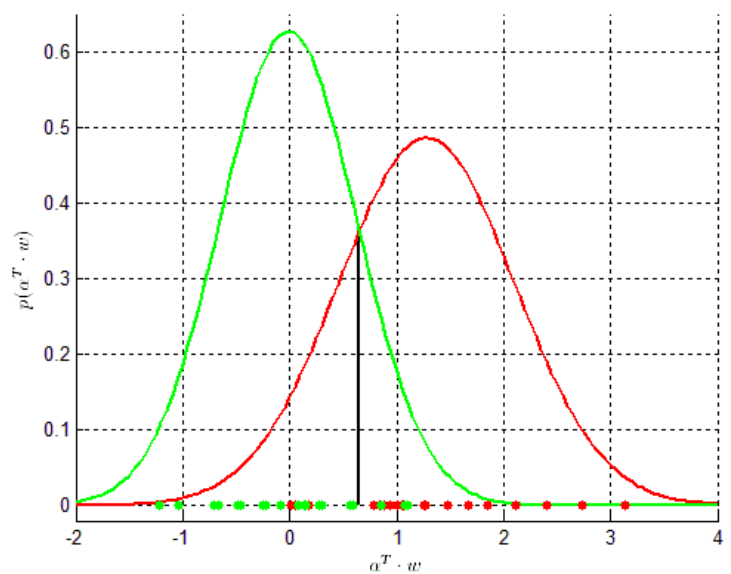

(a)

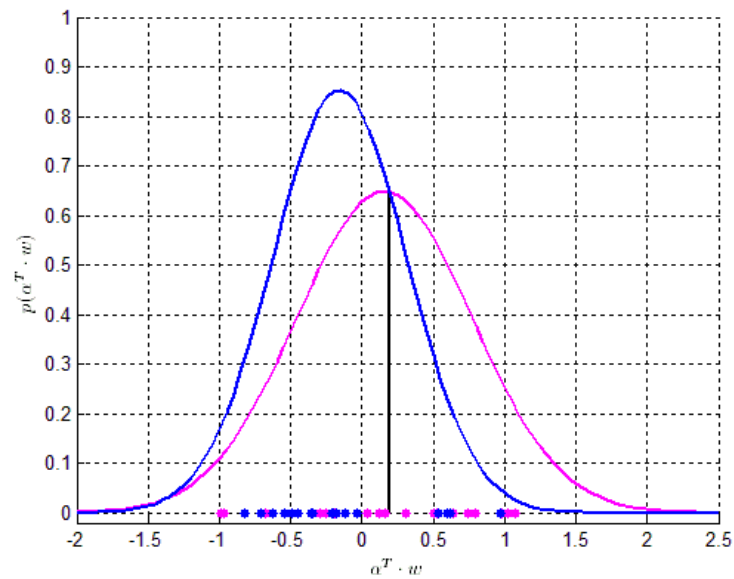

(b)

Fig. 1 Probability distribution of the projections of 3-dimensional MFS exponent of (a) RR signals for young (green) and elderly (red) (b) IBI signals for young (blue) and elderly (magenta). Exponents for elderly people have larger mean and spread. 


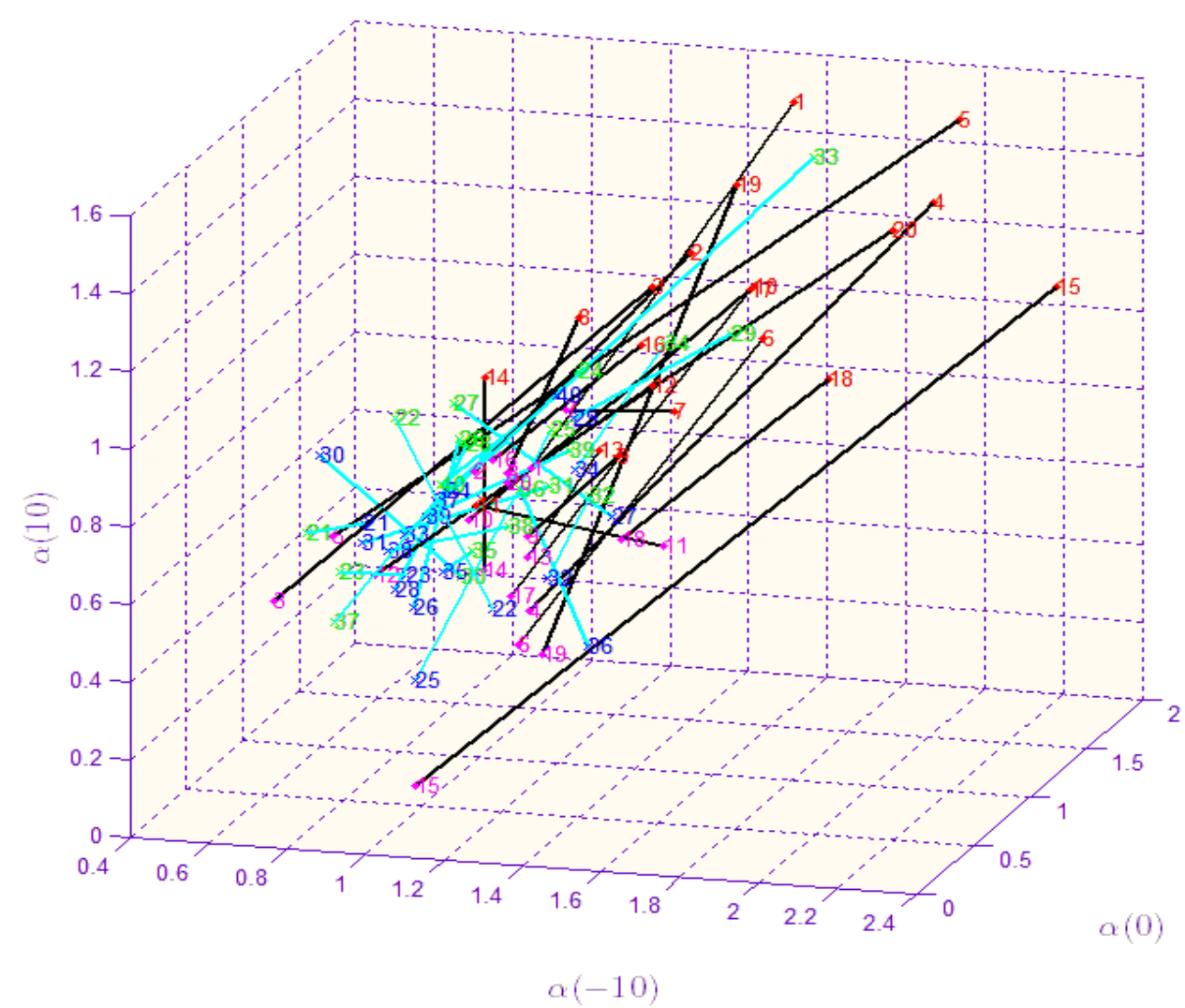

Fig 2. Feature space of lines connecting the Multifractal scale exponents of Heartbeat and Respiration Dynamics. The Heartbeat interval exponents of elderly [68-81] is marked in red, young [21-32] is marked in green. The Interbreath interval exponents of elderly is marked in magenta, young is marked in blue.

\begin{tabular}{ccccc}
\hline$N$ & age(gender) & $R$ & $\theta$, deg & $\phi$, deg \\
\hline 1 & $77(\mathrm{~F})$ & 1.13 & 42 & 50 \\
2 & $73(\mathrm{~F})$ & 0.8 & 55 & 52 \\
3 & $73(\mathrm{M})$ & 1.24 & 56 & 43 \\
4 & $81(\mathrm{M})$ & 1.5 & 55 & 53 \\
5 & $76(\mathrm{M})$ & 1.91 & 62 & 42 \\
6 & $74(\mathrm{~F})$ & 1 & 48 & 53 \\
7 & $68(\mathrm{M})$ & 0.3 & 97 & 42 \\
8 & $73(\mathrm{~F})$ & 0.46 & 43 & 53 \\
9 & $71(\mathrm{M})$ & 0.45 & 76 & 44 \\
10 & $71(\mathrm{~F})$ & 0.95 & 58 & 72 \\
11 & $73(\mathrm{M})$ & 0.47 & 79 & 76 \\
12 & $75(\mathrm{~F})$ & 0.86 & 62 & 47 \\
13 & $85(\mathrm{~F})$ & 0.32 & 32 & -172 \\
14 & $70(\mathrm{~F})$ & 0.51 & 36 & 44 \\
15 & $83(\mathrm{M})$ & 2.02 & 56 & 19 \\
16 & $70(\mathrm{M})$ & 0.51 & 64 & 105 \\
17 & $77(\mathrm{M})$ & 1 & 48 & 37 \\
18 & $71(\mathrm{M})$ & 0.66 & 59 & -133 \\
19 & $77(\mathrm{M})$ & 1.27 & 31 & 140 \\
20 & $73(\mathrm{~F})$ & 1.15 & 61 & -175 \\
\hline mean & $73.7 \pm 3.6$ & $0.93 \pm 0.49$ & $56.6 \pm 16$ & $41 \pm 53$ \\
\hline & & $(\mathrm{a})$ & &
\end{tabular}

\begin{tabular}{ccccc}
\hline$N$ & age(gender) & $R$ & $\theta$, deg & $\phi$, deg \\
\hline 21 & $23(\mathrm{~F})$ & 0.15 & 90 & -133 \\
22 & $28(\mathrm{~F})$ & 0.57 & 45 & 140 \\
23 & $34(\mathrm{M})$ & 0.16 & 89 & -175 \\
24 & $31(\mathrm{M})$ & 0.47 & 57 & 52 \\
25 & $23(\mathrm{M})$ & 0.71 & 28 & 26 \\
26 & $30(\mathrm{M})$ & 0.43 & 23 & 67 \\
27 & $21(\mathrm{M})$ & 0.57 & 70 & 151 \\
28 & $30(\mathrm{~F})$ & 0.4 & 29 & 42 \\
29 & $32(\mathrm{~F})$ & 0.52 & 75 & 57 \\
30 & $21(\mathrm{~F})$ & 0.53 & 114 & -28 \\
31 & $23(\mathrm{~F})$ & 0.49 & 77 & 33 \\
32 & $23(\mathrm{M})$ & 0.23 & 36 & 55 \\
33 & $28(\mathrm{~F})$ & 1.4 & 53 & 43 \\
34 & $27(\mathrm{~F})$ & 0.39 & 42 & 50 \\
35 & $25(\mathrm{~F})$ & 0.09 & 65 & 50 \\
36 & $26(\mathrm{M})$ & 0.42 & 32 & 151 \\
37 & $31(\mathrm{M})$ & 0.43 & 124 & -117 \\
38 & $21(\mathrm{M})$ & 0.35 & 72 & -13 \\
39 & $21(\mathrm{~F})$ & 0.38 & 67 & 28 \\
40 & $21(\mathrm{M})$ & 0.38 & 130 & -178 \\
\hline mean & $27.3 \pm 2.4$ & $0.46 \pm 0$. & $66.4 \pm 31$ & $15 \pm 97$ \\
\hline & & & &
\end{tabular}

(a)

Table 1. Distance and spherical angles between multifractal spectrum exponents of heartbeat and respiration dynamics for a) elderly and b) young people. Mean and standard deviation were also computed. 
The direction of the lines connecting HRV and IBI exponents reveals the presence of a uniform shift of fractal spectrum point from respiration to cardiac dynamics directed towards higher dimensions in all degree of fluctuations (table 1). This is consistent with the fact that for elderly subjects, long-range correlation is degraded and there is less memory in heartbeat dynamics[16].

\section{Conclusions}

In this study we investigated the relationship of the heartbeat dynamics and respiration dynamics for 20 elderly and 20 young subjects. We aimed to know if there is a nonlinear relationship between the fluctuations in heartbeat and respiration, using cardiac interbeat interval and interbreath interval as materials. We also wished to determine the effect of aging in this relationship.

The results showed the evidence of non-linear relationship between the heartbeat and respiration dynamics for young subjects, and an evidence for a non- relationship for the elderly subjects. We also saw a uniform shifting of dimensions for elderly subjects, signifying less correlation especially for heartbeat dynamics.

For the further work we shall analyze the joint distribution of the distance and angle of the connecting line of cardiorespiratory MFS and observe if the groups are clearly separated or not.

Also a non-linear model will be set up to predict IBI points using RR signals and vice-versa, using feed-forward neural networks as the model.

\section{References}

[1] F. M. Maria Vittoria Pitzalis, Francesco Massari, Andrea Passantino, Roberto Colombo, Antonella Mannarini, Cinzia Forleo, Paolo Rizzon, "Effect of respiratory rate on the relationships between RR interval and systolic blood pressure fluctuations: a frequency-dependent phenomenon," Cardiovascular Research Oxfor Journal, vol. 2, pp. 332-339, 1998.

[2] P. Calabrese, et al., "Cardiorespiratory interactions during resistive load breathing," $\mathrm{Am}$ Journal of Physiology, vol. 279, pp. R2208-2213, 2000.

[3] JD Schipke, et al., "Effect of Respiration Rate on short-term Heart Rate Variability," Journal of Clinical and Basic Cardiology, vol. 2, pp. 92-95, 1995.

[4] N. Iyengar, et al., "Age-related alterations in the fractal scaling of cardiac interbeat interval dynamics," American Journal of Physiology, Regulatory, Integrative and Comparative Physiology, vol. 217, 1996.

[5] C.-K. Peng, et al., "Quantifying fractal dynamics of human respiration: age and gender effects," Annals of Biomedical Engineering, vol. 30(5), pp. 683-692, 2002.

[6] S. M. B. Paul J. Fadel, Shaun W. Phillips and Gerard L. Gebber, "Fractal fluctuations in Human Respiration," J Appl Physiol, 2004.

[7] B. J. West, et al., "The independently fractal nature of respiration and heart rate during exercise under normobaric and hyperbaric conditions," Respiratory Physiology \& Neurobiology, vol. 145, 2005.

[8] J.-O. F. Yoshiharu Yamamoto, RIchard Hughson, "On the Fractal Nature of Heart Rate Variability in Humans: Effects of Respiratory Sinus Arrhythmia," 1995.

[9] A. L. a. A. Goldberger, L. A. N. and Glass, L. and Hausdorff, J. M. and Ivanov, P. Ch. and Mark, R. G. and Mietus, J. E. and Moody, G. B. and Peng, C.-K. and Stanley, H. E. PhysioBank, PhysioToolkit, and PhysioNet $\}$ : Components of a New Research Resource for Complex Physiologic Signals [Online].

[10] E. Billauer. peakdet: Peak detection using $M A T L A B$.

[11] J. E. M. C.-K. Peng, Y. Liu, C. Lee, J.M. Haursdorff, H. E. Stanley, A. L. Goldberger, L.A. Lipsitz. (2002) Quantifying Fractal Dynamics of Human Respiration: Age and Gender Effects. Annals of Biomedical Engineering. 683-692.

[12] A. A. Lenskiy, et al., "Multifractal scaling in cardiac interbeat interval dynamics of elderly and young healthy people," In preparation.

[13] C.-K. Peng, et al., "Finite-size effects on longrange correlations: Implications for analyzing DNA sequences," Phys. Rev. E, vol. 47, pp. 37303733, 1993.

[14] Jan W. Kantelhardt, et al., "Multifractal Detrended Fluctuation Analysis of Nonstationary Time Series," Physica A: Statistical Mechanics and its Applications, vol. 316, pp. 87-114, 2002.

[15] K. Falconer, "Hausdorff measure and dimension," in Fractal Geometry: Mathematical Foundations and Application., 2nd ed: John Wiley \& Sons, 2003.

[16] P. C. Ivanov, "Scaling behaviour of heartbeat intervals obtained by wavelet-based time-series analysis," Nature, vol. 383, pp. 323-327, 1996. 